\section{Emergency medical services - poor response time in the rural Eastern Cape}

To the Editor: Emergency medical services (EMS) throughout South Africa are of unequal quality owing to historical population inequalities and under-resourced EMS in rural areas. ${ }^{1}$ There are no data regarding the quality of ambulance services in the rural Eastern Cape. The assessment of EMS is not easy, but an assessment tool has been established. ${ }^{2}$ We prospectively audited the response time to ambulance requests from a community health centre in the rural Eastern Cape.

Ngcwanguba Health Care Centre has a catchment population exceeding 60000 and is staffed by one full-time doctor and 10 professional nurses. Ambulances are stationed at Mthatha and have to use an $80 \mathrm{~km}$-long tarred but severely pot-holed road. They return on this road to the nearest tertiary referral hospitals or take a gravel road of $25 \mathrm{~km}$ to the district hospital.

All calls for ambulances made in the period from 1 July 2009 to 31 September 2009 to refer patients to one of the two referral hospitals were included. No calls were done by the doctor.

\section{Results}

Table I summarises the number of ambulance arrivals and the time involved, over the review period.

\section{Discussion}

The Department of Health in Eastern Cape Province recently published a poster with 'generic standards' that states: 'Emergency medical services (EMS) - All citizens in need of emergency services will be attended to with courtesy by qualified personnel and a fully equipped ambulance within 1 hour in the rural areas and 45 minutes in urban at all times. This standard is clearly failed by the local EMS provider, who achieved this aim in only $3.3 \%$ of ambulance requests. The average response time of an arriving ambulance of almost 4 hours is unacceptably high. The $16.7 \%$ of EMS cases that did not arrive at all is worrying, and a challenge for health carers remaining with the critically ill patient.

An analysis of perinatal deaths in the district hospital showed that, among the 8 avoidable factors, the unavailability and delay of transport were the most common. ${ }^{3}$ Sixteen years after the end of the apartheid era, South Africa shows an unacceptable EMS response time (which is an important and basic component of health care service $^{4}$ ) in the former Transkei. EMS services must be assessed and improvement wisely planned to ensure the basic rights of government sector patients. Although a new EMS station was begun to be built next to the Ngcwanguba Health Care Centre, there has been no progress for a year.

\section{E Meents}

Ngcwanguba Community Health Care Centre

Eastern Cape Province

housearzt@gmx.de

\section{T Boyles}

Division of Clinical Pharmacology

University of Cape Town

Table I. Response times and number of arrivals

\begin{tabular}{|c|c|c|c|c|c|c|c|c|}
\hline Response time (h) & $<1$ & $1-2$ & $2-3$ & $3-4$ & $4-5$ & $5-6$ & $6-12$ & $\begin{array}{l}\text { Failed to } \\
\text { arrive }(>12 \mathrm{~h})\end{array}$ \\
\hline Arrivals & $1(3.3 \%)$ & $4(13.3 \%)$ & $6(20 \%)$ & $4(13.3 \%)$ & $4(13.3 \%)$ & $4(13.3 \%)$ & $2(6.7 \%)$ & $5(16.7 \%)$ \\
\hline
\end{tabular}

